\title{
Stress-Induced Alterations in Prefrontal Cortical Dendritic Morphology Predict Selective Impairments in Perceptual Attentional Set-Shifting
}

\author{
Conor Liston, ${ }^{1}$ Melinda M. Miller, ${ }^{1}$ Deena S. Goldwater ${ }^{2}$ Jason J. Radley, ${ }^{3}$ Anne B. Rocher, ${ }^{2}$ Patrick R. Hof, ${ }^{2}$ \\ John H. Morrison, ${ }^{2}$ and Bruce S. McEwen ${ }^{1}$ \\ ${ }^{1}$ Laboratory of Neuroendocrinology, The Rockefeller University, New York, New York 10021, 2Department of Neuroscience, Mount Sinai School of \\ Medicine, New York, New York 10029, ${ }^{3}$ Laboratory of Neuronal Structure and Function, The Salk Institute for Biological Studies, La Jolla, California 92186
}

\begin{abstract}
Stressful life events have been implicated clinically in the pathogenesis of mental illness, but the neural substrates that may account for this observation remain poorly understood. Attentional impairments symptomatic of these psychiatric conditions are associated with structural and functional abnormalities in a network of prefrontal cortical structures. Here, we examine whether chronic stress-induced dendritic alterations in the medial prefrontal cortex (mPFC) and orbital frontal cortex (OFC) underlie impairments in the behaviors that they subserve. After $21 \mathrm{~d}$ of repeated restraint stress, rats were tested on a perceptual attentional set-shifting task, which yields dissociable measures of reversal learning and attentional set-shifting, functions that are mediated by the OFC and MPFC, respectively. Intracellular iontophoretic injections of Lucifer yellow were performed in a subset of these rats to examine dendritic morphology in layer II/III pyramidal cells of the MPFC and lateral OFC. Chronic stress induced a selective impairment in attentional set-shifting and a corresponding retraction (20\%) of apical dendritic arbors in the MPFC. In stressed rats, but not in controls, decreased dendritic arborization in the $\mathrm{mPFC}$ predicted impaired attentional set-shifting performance. In contrast, stress was not found to adversely affect reversal learning or dendritic morphology in the lateral OFC. Instead, apical dendritic arborization in the OFC was increased by $43 \%$. This study provides the first direct evidence that dendritic remodeling in the prefrontal cortex may underlie the functional deficits in attentional control that are symptomatic of stress-related mental illnesses.
\end{abstract}

Key words: stress; attention; anterior cingulate cortex; lateral orbitofrontal cortex; cell loading; dendrite

\section{Introduction}

Stressors of diverse types elicit a stereotyped set of neuroendocrine and autonomic adaptive responses that serve to mobilize bodily resources that facilitate coping with acute emergencies. Despite these benefits, stressors that are either prolonged or extreme may have cumulative and deleterious cardiovascular, metabolic, and immunosuppressive consequences, a phenomenon known as allostatic load (McEwen, 1998). Stressful life events may also predispose susceptible individuals to a variety of psychiatric conditions, including depression, post-traumatic stress disorder, and other anxiety disorders (Sapolsky, 1996; Heim et al., 1997; McEwen, 1998; Caspi et al., 2003). Increasing evidence suggests that the prefrontal cortex may play a critical role in these psychiatric disorders. Attentional impairments symptomatic of these conditions are associated with structural and functional

Received March 19, 2006; revised May 25, 2006; accepted May 27, 2006.

This work was supported in part by National Institute of Mental Health Grants 5 P50 MH58911 and MH 41256 to B.S.M. C.L. is supported by National Institutes of Health Medical Scientist Training Program Grant GM 07739, a W. M. Keck Foundation Medical Scientist Fellowship, and a Paul and Daisy Soros Fellowship. We thank W. G. M. Janssen, E. Bloss, and A. Wang for technical assistance with perfusions and imaging.

Correspondence should be addressed to Conor Liston, Laboratory of Neuroendocrinology, The Rockefeller University, 1230 York Avenue, Box 165, New York, NY 10021. E-mail: cliston@rockefeller.edu.

DOI:10.1523/JNEUROSCI.1184-06.2006

Copyright $\odot 2006$ Society for Neuroscience $\quad$ 0270-6474/06/267870-05\$15.00/0 anomalies in a circuit of prefrontal cortical structures (Cohen and Servan-Schreiber, 1992; Drevets et al., 1997; Casey et al., 2002; Rauch et al., 2003).

Despite these advances, relatively little is known about the neural substrates that underlie attentional deficits in stressrelated psychiatric illnesses. Some progress has been made in elucidating the cellular morphologic changes in the mPFC after repeated emotional stress in rats. Such work has demonstrated that repeated restraint stress induces retraction and debranching of apical dendrites (Radley et al., 2004, 2006; Cook and Wellman, 2004) and axospinous synapse loss in the rat mPFC (Radley et al., 2006).

The functional consequences of these morphological alterations remain unclear. The rodent $\mathrm{mPFC}$ is believed to play a critical role in modulating attention. Birrell and Brown (2000) tested rats with mPFC lesions on a perceptual attentional setshifting task that yields dissociable measures of discrimination learning, reversal learning, and attention shifting. They found that $\mathrm{mPFC}$ lesions impaired perceptual attentional set-shifting but not other forms of learning. In contrast, a later study demonstrated that orbitofrontal cortical lesions impair reversal learning but not attentional set-shifting (McAlonan and Brown, 2003). Collectively, these studies raise the possibility that stress-induced morphologic alterations may impair attentional control and that 


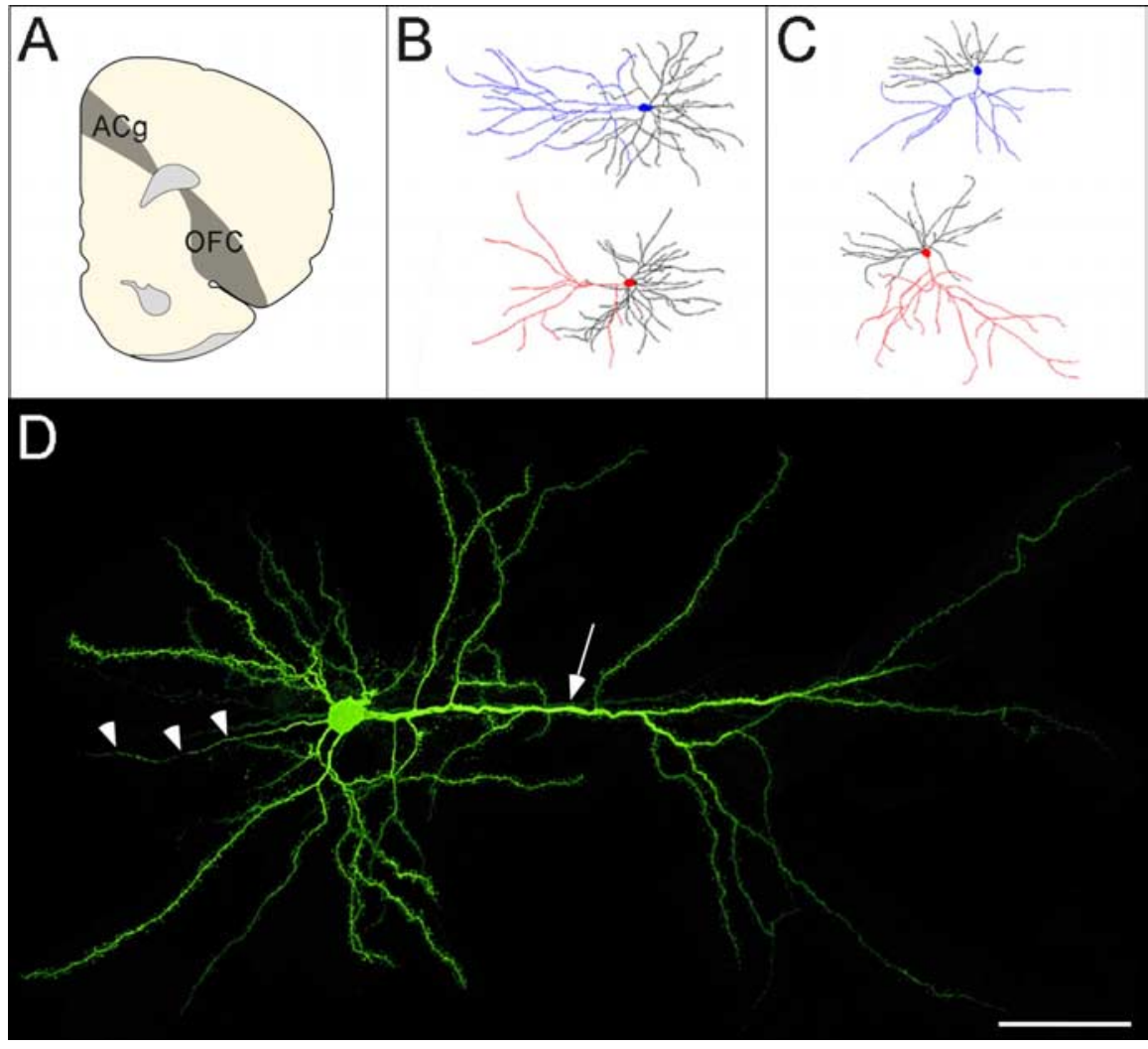

Figure 1. A, Coronal hemisection of the prefrontal cortex [bregma $3.20 \mathrm{~mm}$, adapted from Swanson (1992)] depicting ACg and lateral $\mathrm{OFC}$ regions of interest. $\boldsymbol{B}, \boldsymbol{C}$, Dendritic reconstructions of neurons from $\mathrm{ACg}(\boldsymbol{B})$ and lateral $\mathrm{OFC}(\boldsymbol{C})$, with apical dendrites extending from the soma toward the pial surface at right and the axon (arrowheads) extending to the left. Scale bar, $50 \mu \mathrm{m}$. highlighted in blue (controls) and red (stressed). D, A typical pyramidal neuron from lateral OFC, with the apical dendrite (arrow)

secutive correct trials in an apparatus adapted from those described in Birrell and Brown (2000) and Fox et al. (2003). Testing started with a simple discrimination (SD) in which the rat discriminated between either two odors or two digging media, one of which predicted the food reward. Next, in a compound discrimination $(\mathrm{CD})$, a new dimension was introduced, but the positive stimulus was the same as in the $\mathrm{SD}$. This was followed by an intradimensional attentional shift (IDS), in which two new exemplars from each dimension were introduced, but the task-relevant dimension was the same as in the SD and CD. Next, the IDS was reversed, such that the formerly negative stimulus became the positive stimulus. Finally, an extradimensional attentional shift (EDS) occurred. Here, two new exemplars from each dimension were introduced, and the formerly taskirrelevant dimension became the relevant one. A typical task sequence and a description of the test stimuli are included in supplemental Table 1 (available at www.jneurosci.org as supplemental material). In all other respects, the testing, habituation, and counterbalancing procedures were similar to those described in Fox et al. (2003).

Analysis of prefrontal dendritic morphology. On the day after behavioral testing, 12 rats (six stressed, six controls) were given a euthanizing dose of Nembutal and perfused transcardially with $4 \%$ paraformaldehyde in $0.1 \mathrm{~m}$ phosphate buffer. Brains were dissected and postfixed for $2 \mathrm{~h}$ in the same fixative. To minimize bias, each brain was coded before perfusion, and the code was not broken until the morphometric analyses were completed.

the functional consequences of these effects in mPFC and OFC could be dissociated based on their differential roles in attentional processing.

Thus, the aim of the present study was to examine the relationship between stress-related effects on prefrontal cortical dendritic morphology and attentional control. After $21 \mathrm{~d}$ of repeated restraint stress, 24 rats (12 stressed, 12 controls) were tested on a perceptual attentional set-shifting task. We then performed intracellular iontophoretic injections of Lucifer yellow in a subset of these rats to examine dendritic morphology in layer II/III pyramidal cells of the lateral OFC and the anterior cingulate $(\mathrm{ACg})$ region of the $\mathrm{MPFC}$.

\section{Materials and Methods}

Animals. Twenty-four male Sprague Dawley rats (Charles River, Wilmington, MA) weighing 250-280 g at the start of the experiment were pair-housed and maintained on a $12 \mathrm{~h}$ light/dark schedule (lights on from 7:00 A.M. to 7:00 P.M.). All rats were handled daily for $7 \mathrm{~d}$ before beginning the experiment. Twelve of these rats were restrained in wiremesh restrainers for $6 \mathrm{~h}$ daily (10:00 A.M. to 4:00 P.M.) for $21 \mathrm{~d}$. Control rats $(n=12)$ and stressed rats $(n=12)$ were housed in separate rooms. During the first $11 \mathrm{~d}$ of the experiment, all rats had access to food and water ad libitum, except during restraint sessions. Thereafter, all rats were maintained on a restricted diet of 15-20 g of food per day, with $85 \%$ of ad libitum body weight serving as a guideline. Water remained available ad libitum throughout the experiment. All procedures were approved by the Rockefeller University Institutional Animal Care and Use Committee.

Behavioral paradigm. Rats can be trained to dig in bowls filled with sawdust to retrieve a food reward (Wood et al., 1999). All rats were tested on a series of five discriminations (supplemental Table 1; available at www.jneurosci.org as supplemental material), to a criterion of six con-
The iontophoretic cell loading procedure was identical to that described in Radley et al. (2006). Briefly, neurons in layer II/III of the anterior cingulate region of $\mathrm{mPFC}$ and lateral OFC were loaded with intracellular iontophoretic injections of 5\% Lucifer yellow (Invitrogen, Eugene, OR). The anterior cingulate cortex was delineated based on criteria described in Radley et al. (2006). The lateral orbitofrontal cortex was delineated by loading cells on the orbital aspect of frontal cortex, dorsal to the olfactory bulb, in coronal sections located $\sim 2-3 \mathrm{~mm}$ anterior to the rostral aspect of the genu of the corpus callosum, where this region occupies the lateral $\sim 60 \%$ of orbital cortex (Swanson, 1992).

Sections were coverslipped under PermaFluor, and cells were reconstructed in three dimensions at $400 \times$ using a Zeiss (Oberkochen, Germany) Axiophot 2 microscope and Neurolucida software (MicroBrightField, Williston, VT). Inclusion in the analysis required that neurons lie within layer II/III of the anterior cingulate or lateral orbitofrontal cortex, exhibit complete filling of dendritic arbors as evidenced by well defined endings, and display pyramidal cell morphology (Radley et al., 2004, 2006).

\section{Results}

After $21 \mathrm{~d}$ of repeated restraint, stressed rats appeared well groomed and healthy such that they were indistinguishable from controls. However, stressed rats weighed significantly less than controls $(t=2.96 ; p=0.007)$, consistent with previous reports (Watanabe et al., 1992; Radley et al., 2004, 2006). Previous studies have confirmed that the $21 \mathrm{~d}$ repeated restraint stress model induces increased plasma corticosterone and increased adrenal weights (Watanabe et al., 1992; Magarinos and McEwen, 1995), so these assays were not performed here.

Effects of stress on dendritic morphology in the ACg and OFC Figure 1 depicts the results of our cell loading procedure, includ- 


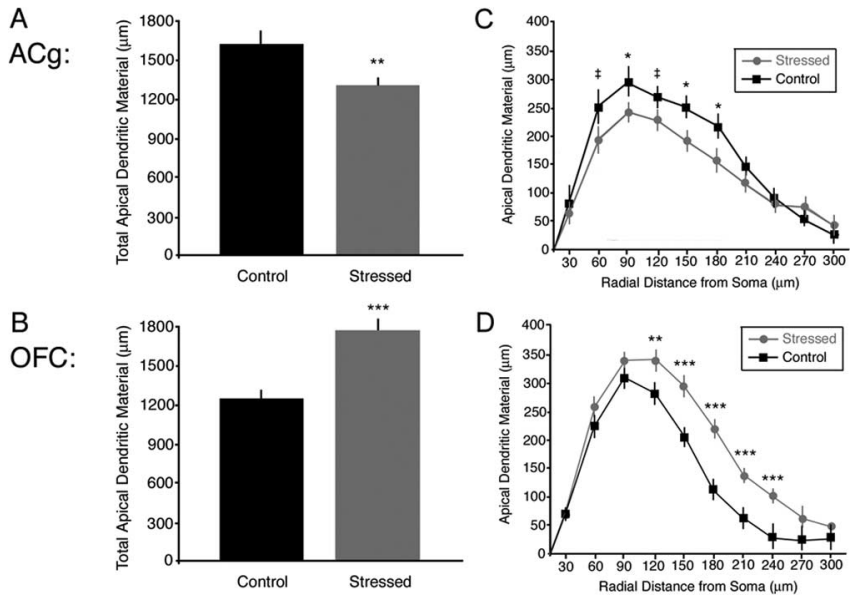

Figure 2. Chronic stress induced contrasting effects on apical dendritic arborization in $\mathrm{ACg}$ and $\mathrm{OFC} . \boldsymbol{A}, \boldsymbol{B}, \operatorname{In} \mathrm{ACg}(\boldsymbol{A})$, total apical dendritic material decreased with stress, whereas a stress-related increase in apical dendritic material was observed in $\mathrm{OFC}(\boldsymbol{B})$. C, D, Sholl analyses. Stress effects on apical dendritic arborization were most pronounced at distances of $90-180$ $\mu \mathrm{m}$ from the soma in $\mathrm{ACg}(\boldsymbol{C})$. In OFC (D), stress affected more distal aspects of the dendrite, with significant increases at $120-240 \mu \mathrm{m}$. Error bars indicate SEM. ${ }^{\ddagger} p<0.10$; ${ }^{*} p<0.05$; ${ }^{* *} p<0.01 ;{ }^{* *} p<0.005$.

ing a typical layer II/III orbitofrontal pyramidal cell and Neurolucida tracings of typical ACg and OFC pyramidal cells from stressed rats and controls. Seventy-two lateral OFC cells (36 stressed, 36 controls) and 54 ACg cells (16 controls, 38 stressed) met the criteria for inclusion in the morphometric analysis.

Repeated restraint stress induced contrasting effects on apical dendrites in the ACg and lateral OFC. As reported previously (Radley et al., 2004), medial frontal apical dendritic material ( $t=$ $2.83 ; p=0.007)$ and branching $(t=1.99 ; p=0.05)$ were reduced by 20 and $11 \%$, respectively, in stressed animals relative to controls (Fig. 2A), whereas basal dendritic material was unaffected $(t=0.41 ; p=0.69)$. A Sholl analysis (Fig. $2 C)$ revealed main effects of stress $\left(F_{(1,52)}=8.17 ; p=0.006\right)$ and radial distance from cell body $\left(F_{(9,468)}=84.69 ; p<0.001\right)$ on apical dendritic material. Post hoc contrasts indicated that the effect of stress was most pronounced at distances of $90(t=2.04 ; p=0.05), 150(t=$ $2.31 ; p=0.03)$, and $180 \mu \mathrm{m}(t=2.32 ; p=0.05)$.

In contrast, stress induced a proliferative effect in the lateral OFC that extended to more distal aspects of the apical dendrite. Total apical dendritic material $(t=4.64 ; p<0.001)$ and branching $(t=3.64 ; p=0.001)$ increased by 43 and $36 \%$, respectively, in stressed animals relative to controls (Fig. $2 \mathrm{~B}$ ). As in the ACg, no significant differences were observed for basal dendritic material $(t=0.94 ; p=0.35)$. A Sholl analysis (Fig. 2D) revealed main effects of stress $\left(F_{(1,70)}=18.50 ; p<0.001\right)$ and radial distance $\left(F_{(9,630)}=213.88 ; p<0.001\right)$ on apical dendritic material. Post hoc contrasts revealed stress-related increases in apical dendritic material at distances of $120-240 \mu \mathrm{m}$ from the cell body $(t>2.71$; $p=0.009)$. A significant region by group by distance interaction $\left(F_{(9,1098)}=4.96 ; p<0.001\right)$ confirmed that stress affected more distal aspects of the apical dendrite in the OFC relative to the $\mathrm{ACg}$.

\section{Effects of stress on attentional set shifting}

Repeated restraint stress induced a selective impairment in extradimensional attentional set shifting, but not discrimination or reversal learning (Fig. 3A). An overall ANOVA with task phase as a within-subjects factor and group (stressed or control) and initial relevant dimension (medium or odor) as between-subjects factors revealed main effects of task phase $\left(F_{(4,80)}=26.6 ; p<\right.$ $0.001)$ and group $\left(F_{(1,20)}=7.63 ; p=0.01\right)$ and a task phase by group interaction $\left(F_{(4,80)}=5.85 ; p<0.001\right)$. Post hoc analyses demonstrated that this interaction was driven by the EDS phase, with stressed rats significantly impaired relative to controls $(t=$ $3.51 ; p=0.002)$ and no significant between-group differences on any other task phase $(t<1.33 ; p>0.20)$. Importantly, stressed rats were not impaired on reversal learning $(t=0.43 ; p=0.67)$, which in control rats was equivalent in difficulty to the EDS. This indicates that the effect of stress was specific to attention shifting and did not reflect a generalized learning impairment. No other main effects or interactions were observed in this analysis $(p>$ $0.60)$.

The validity of the task design was confirmed with a separate within-group ANOVA examining control rats only, with task phase as a within-subjects factor and initial relevant dimension (medium or odor) as a between-subjects factor. As expected, there was a main effect of task phase $\left(F_{(4,40)}=8.73 ; p<0.001\right)$. The EDS was significantly more difficult than the IDS $(t=3.65$; $p=0.001)$, confirming the validity of the extradimensional attention shifting manipulation. There was no main effect of initial relevant dimension or interaction of this effect with other factors $(p>0.40)$, indicating that the odor-to-medium and mediumto-odor shifts were equivalent in difficulty.

\section{Analysis of individual differences in morphology and attention shifting}

To assess whether intersubject morphologic variation predicted behavioral performance, we computed mean apical dendritic lengths for each region in each subject. Rats with the largest stress-related morphologic alterations showed the greatest impairments in attention shifting. In the stressed group, rats with the greatest stress-related retractions of $\mathrm{ACg}$ apical dendrites tended to show larger attention-shifting impairments (Fig. 3B) $(r=-0.74 ; p=0.09)$. No association between ACg arborization and attention was detectable in controls $(r=-0.54 ; p=0.35)$. To confirm these results, we classified stressed and control rats into four groups based on median splits of their respective ACg arbors. ANOVA confirmed a main effect of this grouping on EDS performance $\left(F_{(3,8)}=7.35 ; p=0.01\right)$. Stressed rats with the largest $\mathrm{ACg}$ morphologic alterations were significantly impaired on the EDS phase relative to rats in the other three groups (Fig. $3 C$ ) $(t>3.43 ; p<0.026)$, whereas stressed rats with lesser morphologic alterations performed equivalently to controls on the EDS phase $(t<0.38 ; p>0.72)$. In contrast, ACg arborization in control rats had no effect on EDS performance $(t=0.25 ; p=$ 0.82 ), in accord with the correlations reported above.

\section{Discussion}

Our results indicate that chronic stress induces contrasting morphologic effects in medial and lateral orbitofrontal cortices. In accord with previous reports (Radley et al., 2004, 2006), chronic stress was associated with a $20 \%$ decrease in apical dendritic material in the anterior cingulate region of mPFC. In contrast, stress induced a $43 \%$ increase in apical dendritic material in layer II/III pyramidal cells of lateral OFC. Accordingly, stress selectively impaired extradimensional attention shifting, which depends on mPFC function, but not reversal learning, an OFC-dependent function.

Our morphologic results from the ACg are in agreement with several previous studies using the Golgi impregnation and iontophoretic cell-loading techniques (Radley et al., 2004, 2006; Cook and Wellman, 2004). In separate studies using iontophoretic cell 

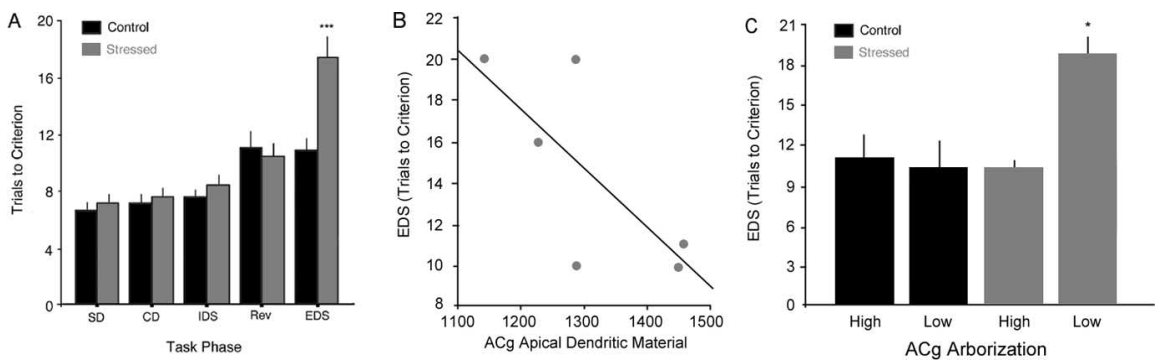

Figure 3. $A$, Chronic stress selectively impaired EDS, leaving discrimination learning (SD, CD) and reversal learning (Rev) unaffected. $\boldsymbol{B}$, Stress-related decreases in ACg apical dendritic material predicted attentional impairments in the EDS phase ( $r=$ $-0.74 ; p=0.09$ ). No association was observed in controls (data not shown). $\boldsymbol{C}$, Stressed rats and controls were divided into four groups based on median splits of their respective ACg apical dendritic lengths. Stressed rats (gray) with the largest ACg morphologic effects ("low ACg arborization") showed significant attention shifting impairments, whereas stressed rats with minimal morphologic changes ("high arborization") performed comparably with controls. No association between ACg arborization and attention shifting was observed in controls. Error bars indicate SEM. ${ }^{*} p<0.05 ;{ }^{* * *} p<0.005$.

loading, Radley and colleagues (2004, 2006) observed decreases in medial prefrontal apical dendritic material of $20-22 \%$, in close accord with the $20 \%$ reduction reported here. The design of these studies was identical to that used here, except that rats in our study were maintained on a restricted diet for the last $10 \mathrm{~d}$ of the experiment. The fact that our results are in close agreement suggests that dietary restriction did not confound our findings. Indeed, numerous reports indicate that mild dietary restriction can prolong life span, ameliorate age-related declines in physiologic functions, and reduce the incidence of autoimmune disease (Weindruch and Walford, 1988; Kubo et al., 1992). The fact that these results were highly replicable also highlights the utility of iontophoretic cell loading, which facilitates more precise morphometry relative to the Golgi technique by ensuring that cells are completely filled and by eliminating overlapping dendritic fields.

Although several studies have reported decreased apical dendritic arborization in the ACg with stress, our OFC morphology findings are, to our knowledge, the first report of stress-related increases in arborization in any region of the frontal cortex. Two previous studies reported contrasting effects of chronic stress on hippocampal and amygdaloid pyramidal cells, with cells of the basolateral amygdala undergoing marked increases in apical dendritic arborization and spine density (Vyas et al., 2002). The mechanisms by which chronic stress induces contrasting morphologic effects in these two regions remain unclear. At the molecular level, previous studies indicate that glucocorticoids and excitatory neurotransmitters may act in concert in the hippocampus to induce dendritic atrophy (Magarinos and McEwen, 1995). Glucocorticoids also act to enhance calcium currents in hippocampal pyramidal cells, which can induce dendritic remodeling, and it has been suggested that the contrasting effects of stress on hippocampal and amygdalar plasticity may be attributable to differences in the spatiotemporal dynamics of intracellular calcium concentrations (Kerr et al., 1992; McEwen, 2000). Neuronal cell adhesion molecules (Sandi, 2004) and serotonergic neuromodulatory influences (Conrad et al., 1996; Stutzmann et al., 1998) may also play a prominent role. Similar pharmacologic manipulations may shed light on the mechanisms by which stress induces contrasting effects in the ACg and lateral OFC.

Given these contrasting morphologic effects, the selective impairment in attention shifting can be easily understood in the context of previous lesion studies. In separate studies, Brown and colleagues demonstrated a double dissociation by which $\mathrm{mPFC}$ lesions impair attention shifting but not reversal learning (Birrell and Brown, 2000), whereas OFC lesions impair reversal learning but not attention shifting (McAlonan and Brown, 2003). Our results confirm that chronic stress reduces apical dendritic material by $\sim 20 \%$, which, in combination with a $16 \%$ reduction in spine density (Radley et al., 2006), may lead to a $33 \%$ reduction in axospinous input to layer II/III apical dendrites in the ACg. Layer II/III pyramidal cells are both the origin and target of long-range corticocortical connections and are likely to play an important computational role in cognitive (e.g., attentional) functions mediated by a distributed network of structures (Dehaene et al., 1998). As such, it is likely that a stress-related reduction in axospinous input to these cells contributes to the selective impairment in attention shifting (Fig. $3 A$ ). This hypothesis is in accord with a recent report demonstrating an association between corticosteroid-induced atrophy of mPFC layer II and impairments in behavioral flexibility (Cerqueiras et al., 2005). It is also supported by our observation that the magnitude of ACg morphologic alterations predicted the degree of attentional impairment (Fig. $3 B-C$ ). Interestingly, this correlation was specific to stressed rats: ACg arborization was not associated with task performance in controls, and only rats with the largest stress-induced retractions of ACg arbors showed significant attentional impairments, suggesting that the circuitry may be resilient to smaller variations in axospinous input.

Although earlier lesion studies provide a framework for understanding the effects reported here, a few anatomical distinctions are worth noting. In particular, the lesions in the Birrell and Brown (2000) study extended into regions of both the dorsal $\mathrm{mPFC}$, including the $\mathrm{ACg}$ and prelimbic cortex, and the ventral (infralimbic) $\mathrm{mPFC}$ in all animals, so the $\mathrm{mPFC}$ contribution to attention shifting cannot be localized to the ACg based on these results. However, converging findings from a variety of studies (for review, see Heidbreder and Groenewegen, 2003) indicate that the dorsal mPFC shares salient cytoarchitectonic, neurochemical, and anatomical characteristics with the primate lateral prefrontal cortex, which has been implicated in attentional setshifting in closely related paradigms (Dias et al., 1996; Liston et al., 2006). Accordingly, Nicolle and Baxter (2003) reported that age-related decreases in ACg kainate binding correlated with attention shifting impairments. These results support the idea that the rodent dorsal $\mathrm{mPFC}$, rather than infralimbic cortex, may play a critical role in attentional control and other functions ascribed to the lateral PFC in primates. They are also consistent with our hypothesis that stress-related reductions in axospinous input to the ACg may contribute to impairments in attentional control.

In contrast, enhanced OFC arborization was not associated with a significant enhancement in reversal learning. This may be attributable in part to a ceiling effect, whereby the speed of reversal learning observed in control rats cannot be substantially improved on by enhanced OFC inputs. Alternatively, it is likely that attention shifting and reversal learning are both mediated by multiple structures acting in concert (O'Reilly et al., 2002; Fox et al., 2003; Liston et al., 2006), and our observations may reflect alterations in other regions not examined here. Future work will target additional regions of association cortex and examine how they interact to mediate these behaviors. Likewise, our results point to an association between $\mathrm{ACg}$ arborization and attentional 
control, but they do not rule out contributions from other stressdependent factors. For example, some reports suggest that mPFC-dependent cognitive functions may be modulated by stress-related alterations in noradrenergic (Roozendaal et al., 2004) and dopaminergic (Mizoguchi et al., 2000) inputs to the mPFC, which in turn may reflect local postsynaptic structural changes consistent with our results or presynaptic changes specific to the cells that are the source of these projections. In either case, our findings highlight the need for more detailed anatomical and pharmacological studies that could distinguish between these possibilities.

Collectively, our results indicate that chronic stress induces contrasting morphologic effects in the lateral OFC and ACg, which in turn predict the severity of stress-related impairments in attention shifting. They provide direct evidence that prefrontal dendritic remodeling may contribute to the attentional impairments that are symptomatic of depression and anxiety disorders. Thus, they may facilitate future efforts to elucidate the relationship between stress and psychiatric conditions (Sapolsky, 1996; Heim et al., 1997; Caspi et al., 2003) that feature prominent deficits in attentional control, accompanied by structural and functional alterations in prefrontal circuits that regulate attention and affective behaviors (Davidson et al., 2002).

\section{References}

Birrell JM, Brown VJ (2000) Medial frontal cortex mediates perceptual attentional set shifting in the rat. J Neurosci 20:4320-4324.

Casey BJ, Tottenham N, Fossella J (2002) Clinical, imaging, lesion, and genetic approaches toward a model of cognitive control. Dev Psychobiol 40:237-254

Caspi A, Sugden K, Moffitt TE, Taylor A, Craig IW, Harrington H, McClay J, Mill J, Martin J, Braithwaite A, Poulton R (2003) Influence of life stress on depression: moderation by a polymorphism in the 5-HTT gene. Science 301:386-389.

Cerqueiras JJ, Pego JM, Taipa R, Bessa JM, Almeida OFX, Sousa N (2005) Morphological correlates of corticosteroid-induced changes in prefrontal cortex-dependent behaviors. J Neurosci 25:7792-7800.

Cohen JD, Servan-Schreiber D (1992) Context, cortex and dopamine: a Connectionist approach to behavior and biology in schizophrenia. Psychol Rev 99:47.

Conrad CD, Galea LAM, Kuroda Y, McEwen BS (1996) Chronic stress impairs rat spatial memory on the Y-maze and this effect is blocked by tianeptine pre-treatment. Behav Neurosci 110:1321-1334.

Cook SC, Wellman CL (2004) Chronic stress alters dendritic morphology in rat medial prefrontal cortex. J Neurobiol 60:236-248.

Davidson RJ, Pizzagalli D, Nitschke JB, Putnam (2002) Depression: perspectives from affective neuroscience. Annu Rev Psychol 53:545-574.

Dehaene S, Kerszberg M, Changeux JP (1998) A neuronal model of a global workspace in effortful cognitive tasks. Proc Natl Acad Sci USA 95:14529-14534.

Dias R, Robbins TW, Roberts AC (1996) Dissociation in prefrontal cortex of affective and attentional shifts. Nature 380:69-72.

Drevets WC, Price JL, Simpson JR, Todd RD, Reich T, Vannier M, Raichle ME (1997) Subgenual prefrontal cortex abnormalities in mood disorders. Nature 386:824-827.

Fox MT, Barense MD, Baxter MG (2003) Perceptual attentional set-shifting is impaired in rats with neurotoxic lesions of posterior parietal cortex. J Neurosci 23:676-681.

Heidbreder CA, Groenewegen HJ (2003) The medial prefrontal cortex in the rat: evidence for a dorso-ventral distinction based upon functional and anatomical characteristics. Neurosci Biobehav Rev 27:555-579.

Heim C, Owens MJ, Plotsky PM, Nemeroff CB (1997) The role of early adverse life events in the etiology of depression and posttraumatic stress disorder: focus on corticotropin releasing factor. Ann NY Acad Sci 82:194-207.

Kerr DS, Campbell LW, Thibault O, Landfield PW (1992) Hippocampal glucocorticoid receptor activation enhances voltage-dependent $\mathrm{Ca}^{2+}$ conductances: relevance to brain aging. Proc Natl Acad Sci USA 89:8527-8531.

Kubo C, Gajjar A, Johnson BC, Good RA (1992) The effects of dietary restriction on immune function and development of autoimmune disease in BXSB mice. Proc Natl Acad Sci USA 89:3145-3149.

Liston C, Matalon S, Hare TA, Davidson MC, Casey BJ (2006) Anterior cingulate and posterior parietal cortices are sensitive to dissociable forms of conflict in a task-switching paradigm. Neuron 50:643-653.

Magarinos AM, McEwen BS (1995) Stress-induced atrophy of apical dendrites of hippocampal CA3c neurons: involvement of glucocorticoid secretion and excitatory amino acid receptors. Neurosci 69:89-98.

McAlonan K, Brown VJ (2003) Orbital prefrontal cortex mediates reversal learning and not attentional set shifting in the rat. Behav Brain Res 146:97-103.

McEwen BS (1998) Protective and damaging effects of stress mediators. N Engl J Med 338:171-179.

McEwen BS (2000) Effects of adverse experiences for brain structure and function. Biol Psychiatry 48:721-731.

Mizoguchi K, Yuzurihara M, Ishige A, Sasaki H, Chui DH, Tabira T (2000) Chronic stress induces impairment of spatial working memory because of prefrontal dopaminergic dysfunction. J Neurosci 20:1568-1574.

Nicolle MM, Baxter MG (2003) Glutamate receptor binding in the frontal cortex and dorsal striatum of aged rats with impaired attentional setshifting. Eur J Neurosci 18:3335-3342.

O'Reilly RC, Noelle DS, Braver TS, Cohen JD (2002) Prefrontal cortex and dynamic categorization tasks: representational organization and neuromodulatory control. Cereb Cortex 12:246-257.

Radley JJ, Sisti HM, Hao J, Rocher AB, McCall T, Hof PR, McEwen BS, Morrison JH (2004) Chronic behavioral stress induces apical dendritic reorganization in pyramidal neurons of the medial prefrontal cortex. Neuroscience 125:1-6.

Radley JJ, Rocher AB, Miller M, Janssen WGM, Liston C, Hof PR, McEwen BS, Morrison JH (2006) Repeated stress induces dendritic spine loss in the rat medial prefrontal cortex. Cereb Cortex 16:313-320.

Rauch SL, Shin LM, Degal E, Pitman RK, Carson MA, McMullin K, Whalen PJ, Makris N (2003) Selectively reduced regional cortical volumes in post-traumatic stress disorder. NeuroReport 14:913-916.

Roozendaal B, McReynolds JR, McGaugh JL (2004) The basolateral amygdala interacts with the medial prefrontal cortex in regulating glucocorticoid effects on working memory impairment. J Neurosci 24:1385-1392.

Sandi C (2004) Stress, cognitive impairment, and cell adhesion molecules. Nat Rev Neurosci 5:917-930.

Sapolsky RM (1996) Why stress is bad for your brain. Science 273:749-750.

Stutzmann GE, McEwen BS, LeDoux JE (1998) Serotonin modulation of sensory inputs to the lateral amygdala: dependency on corticosterone. J Neurosci 18:9529-9538.

Swanson (1992) Brain maps: structure of the rat brain. New York: Elsevier.

Vyas A, Mitra R, Shankaranarayana Rao BS, Chattarji S (2002) Chronic stress induces contrasting patterns of dendritic remodeling in hippocampal and amygdaloid neurons. J Neurosci 22:6810-6818.

Watanabe Y, Gould E, McEwen BS (1992) Stress induces atrophy of apical dendrites of hippocampal pyramidal neurons. Brain Res 588:341-345.

Weindruch R, Walford RL (1988) The retardation of aging and disease by dietary restriction. Springfield, IL: Charles C. Thomas.

Wood ER, Dudchenko PA, Eichenbaum H (1999) The global record of memory in hippocampal neuronal activity. Nature 397:613-616. 